\title{
Spectral diffusion on ultralong time scales in low temperature glasses
}

\author{
Peter Neu, David R. Reichman, and Robert J. Silbey \\ Department of Chemistry and Center for Materials Science and Engineering, \\ Massachusetts Institute of Technology, Cambridge, Ma. 02139, USA
}

(appears in Phys. Rev. B)

\begin{abstract}
A dynamical theory is constructed to describe spectral diffusion in glasses in the temperature range near 1 Kelvin on long time scales. The theory invokes interacting tunneling centers (TLS) which provide an excess contribution to the spectral hole width which qualitatively accounts for the deviation from standard logarithmic line broadening observed by Maier et al. [Phys. Rev. Lett. 76, 2085 (1996)]. Alternative explanation schemes of the nonlogarithmic line broadening, avoiding interacting TLS, are discussed. We devise experimental tests which could be used to access the validity of the proposed theories.
\end{abstract}

PACS numbers: 61.43.FS, 78.40.-q

\section{INTRODUCTION}

At low temperatures the thermal, acoustic and optical behavior of glasses differs significantly from that of crystalline solids. As examples note the linear temperature dependence of the specific heat, the pronounced absorption of sound even below $1 \mathrm{~K}$, and the anomalous broadening of spectral holes in the homogeneous line of chromophore molecules. It is known now for more than twenty years that phonons cannot account for these observations; instead localized low-energy excitations in the glass are needed. Since the introduction of the standard tunneling model (STM) by Anderson, Halperin and Varmat and Phillipse, and the experimental obserration of saturability of ultrasound by Hunklinger et al. ${ }^{3}$ and Golding et al.t, it is widely accepted that these lowenergy excitations are two-level tunneling systems (TLS). Denoting the left and right ground state of the doublewell potential by $|L\rangle$ and $|R\rangle$, respectively, the Hamiltonian reads in a Pauli-spin representation

$$
H=-\frac{\Delta}{2} \sigma_{x}-\frac{\epsilon}{2} \sigma_{z},
$$

where $\Delta / \hbar$ is the tunneling frequency, $\epsilon$ the asymmetry energy and $\sigma_{z}=|L\rangle\langle L|-| R\rangle\langle R|$. In the STM the interaction between TLS is neglected, and it is assumed that the tunneling parameters $\Delta, \epsilon$ are random variables with distribution

$$
P^{(1)}(\epsilon, \Delta) d \epsilon d \Delta=\frac{P_{0}}{\Delta} d \epsilon d \Delta, \quad \Delta \geq \Delta_{\min },
$$

(with $P_{0} \approx 0.6 \times 10^{45} \mathrm{~J}^{-1} \mathrm{~m}^{-3}$ in PMMA, a polymer glass). The ensuing constant distribution for the TLSenergy splitting

$$
E=\sqrt{\Delta^{2}+\epsilon^{2}}
$$

explains in particular the linear specific heat. Including relaxation of the TLS via the one-phonon process with rate

$$
R=\left(\frac{\Delta}{E}\right)^{2} R_{\max }
$$

where $\left(x=E / 2 k_{B} T\right)$

$$
R_{\max }(E)=\alpha T^{3} x^{3} \operatorname{coth} x
$$

and

$$
\alpha=\frac{\gamma^{2}\left(2 k_{\mathrm{B}}\right)^{3}}{2 \pi \hbar^{4} \varrho v^{5}}
$$

the model describes most acoustic and optical experiments in glasses satisfactorily. Here, $\gamma$ is the deformation potential energy of the TLS-phonon coupling, $\varrho$ the mass density of the glass, and $v$ the sound velocity.

Recent experiments report a systematic disagreement with the STM. An example is the attenuation of sound below $100 \mathrm{mK}$. The STM predicts a $T^{3}$-increase in contradiction with the experimental $T^{(1-2)}$-law. 5 Deviations from the predicted STM behavior was also observed by Maier, Kharlamov, and Harer in low temperature hole-burning experiments 11,22 They performed hole-burning of a chromophore embedded in PMMA at temperatures around $1 \mathrm{~K}$ up to extremely long times (from $10 \mathrm{~s}$ to 10 days). They found a logarithmic timedependence with a crossover to an algebraic behavior after about $3 \mathrm{~h}$. Though the $\log t$-behavior is in agreement with the STM, the algebraic behavior is not. The authors could fit their data with an ad hoc ansatz

$$
P(\epsilon, \Delta)=P_{0}\left[\frac{1}{\Delta}+\frac{A}{\Delta^{2}}\right], \quad A=\text { constant },
$$

for the TLS-parameter distribution function and the assumption that relaxation occurs via the one-phonon process with the rate (4) -(5). They motivate the distribution function (7) by recent publications focusing on the interaction of TLS in glasses.13 16 Indeed, Burin and Kagan 16 have shown, following earlier ideas of $\mathrm{Yu}$ and Leggett 14 that pairs of interacting TLS do provide a means of constituting a distribution like the second term in Eq. (7) for certain excitations in their energy spectrum. They called 
TLS which are distributed according to the first term in (7) primary $T L S$, and those distributed according to the second term in (7), i.e., pairs of primary TLS, secondary $T L S$. The nice feature of their theory is that the distribution of secondary TLS [second term in Eq. (7)] is derived from primary TLS which are distributed according to the STM [first term in Eq. (7)]. In that sense, Burin and Kagan's theory stays within the framework of the STM, and includes only excitations which have not been considered in the traditional treatment. So far these ideas have been worked out very qualitatively, and with emphasis on experiments in the millikelvin regime. Hence, the question arises whether they apply to the experiment of Maier et al., i.e., for relaxation processes at $1 \mathrm{~K}$ on the time scale between hours and days, or whether an extension of the STM - which would be as phenomenological as the STM - has to be found in order to understand this experiment. It is the purpose of this paper to address this issue by presenting a detailed model that includes TLS-TLS coupling in the manner proposed by Burin and Kagan.

The paper is laid out as folloys; in Sec. II, we analyze the experiment of Maier et al.11.12 and show the achievements and failures of Burin and Kagan's approach for experiments in the Kelvin regime; in Sec. III and IV, we propose a specific model that combines interacting TLS with strong-coupling effects between TLS and phongms in the framework of the theory of Kassner and Silbey 17 ; in Sec. V, we compare the predictions of the new model with the hole-burning data in Ref. 12, and also discuss alternative explanation schemes, which comprise an extension of the STM, and compare with equivalent holeburning measurements in proteins; finally, in Sec. VI, we discuss our results and conclude with a short summary in Sec. VII. The mathematical details are relegated to three Appendices in order not to obscure the basic ideas.

\section{HOLE-BURNING AT ULTRALONG TIMES AND THE BURIN-KAGAN THEORY}

In Ref. 11, 12 photo-chemical hole-burning in PMMA at 1 and $0.5 \mathrm{~K}$ has been performed for extremely long times $t_{\max }=10^{6} \mathrm{~s}$. The authors found a $\log t$-dependence with a crossover to an algebraic behavior after approximately $3 \mathrm{~h}$. The crossover shifts 1 order of magnitude in time from $10^{4}$ to $10^{3} \mathrm{~s}$, if the temperature is increased by a factor of 2 .

A theoretical description of spectraldiffusion in glasses was provided by $\mathrm{Hl}$ and Walkert and Black and Halperins. Reineckel, and later Bai and Fayer10, extended their results to optical experiments. Based on this work the dependence of the hole width $\Gamma(t)$ on the waiting time $t$ is determined by

$$
P(E, r)=\frac{P_{0}}{2 \sqrt{1-r}}\left[\frac{1}{r}+\frac{A}{r^{3 / 2} E}\right],
$$

where

$$
r=R / R_{\max }
$$

according to the following equation:

$$
\begin{aligned}
& \Gamma(t)=\frac{\pi^{2}}{3 \hbar}\langle C\rangle \int_{0}^{\infty} d E \operatorname{sech}^{2} \frac{E}{2 k_{B} T} \int_{0}^{1} d r \frac{\epsilon}{E} P(E, r) \\
& \times\left(1-e^{-r R_{\max } t}\right) .
\end{aligned}
$$

Here, $\langle C\rangle$ is the chromophore-TLS coupling strength. The distribution $P(E, r)$ directly follows from Eq. (7) by using (3)-(5) and (9). With this, we find

$$
\begin{aligned}
\Gamma(t)= & \Gamma\left(t_{0}\right)+\frac{\pi^{2}}{3 \hbar}\langle C\rangle P_{0} \\
& \times\left[k_{B} T \log \frac{t}{t_{0}}+A \sqrt{\alpha T^{3}}\left(\sqrt{t}-\sqrt{t_{0}}\right)\right]
\end{aligned}
$$

with

$$
\alpha=9 \times 10^{9} \mathrm{~K}^{-3} \mathrm{~s}^{-1}
$$

in PMMA.18 To compare with the experiment, we have included in $\Gamma\left(t_{0}\right)$ processes which are faster than the shortest experimental time $t_{0}$, for which a hole broadening can be determined. In Ref. 12(a) a fit to the experimental data provided

$$
A / k_{B} \approx 10^{-7} \mathrm{~K}
$$

at 1 and $0.5 \mathrm{~K}$ with an error of approximately 10\%. Furthermore, the experimental observation of spectral diffusion up to $t_{\max } \sim 10^{6} \mathrm{~s}$ suggests

$$
\Delta_{\min } / k_{B} \lesssim 10^{-8} \mathrm{~K}
$$

according to the relation $t_{\max } \leq 1 / R_{\min }=$ $\left(2 k_{B} T / \Delta_{\min }\right)^{2}\left(1 / \alpha T^{3}\right)$.

Very recently, Burin and Kagan 16 showed that weak TLS-TLS interaction provides ultralow energy excitations. They added to the Hamiltonian (11) an interaction term

$$
H_{\mathrm{TLS}-\mathrm{TLS}}=-\frac{1}{4} \sum_{i j} J_{i j} \sigma_{z}^{i} \sigma_{z}^{j},
$$

where the interaction energy

$$
J_{i j}=\frac{\mu_{i j}}{\left|\mathbf{r}_{i}-\mathbf{r}_{j}\right|^{3}}
$$

falls off with distance $\left|\mathbf{r}_{i}-\mathbf{r}_{j}\right|$ in a manner typical for a dipole-dipole interaction. They assumed that the angular average of the coupling is zero,

$$
\left\langle\mu_{i j}\right\rangle=0
$$

and that the TLS-TLS coupling is weak, i.e.,

$$
P_{0} U_{0} \ll 1
$$


where $U_{0}$ is set by the variance

$$
\left\langle\mu_{i j}^{2}\right\rangle=U_{0}^{2} .
$$

Such an interaction could be mediated by virtual phonon exchange (elastic coupling) or virtual photon exchange (electrostatic coupling) between the TLS. In the former case the energy scale of interaction (19) is easily found to be

$$
U_{0}=\frac{\gamma^{2}}{\pi \varrho v^{2}},
$$

which, indeed, provides $P_{0} U_{0} \ll 1$ for all glasses -in PMMAL: $U_{0} \approx 1 \mathrm{eV} \AA^{3}$ and $P_{0} U_{0} \approx 10^{-4}$. Based on the smallness of $P_{0} U_{0}$, Burin and Kagan proposed that the TLS-TLS coupling in glasses is dominated at low temperatures by an up-down transition (cf. Fig. 1). Such an interaction contains coherence because the up transition of one TLS inevitably has to be followed by a down transition of the coupled TLS. After rotating to the eigenbasis $\left|0_{i}\right\rangle,\left|1_{i}\right\rangle$ of (11) by

$$
\begin{aligned}
& \sigma_{z}^{i}=\left(\epsilon_{i} / E_{i}\right) S_{z}^{i}-\left(\Delta_{i} / E_{i}\right) S_{x}^{i}, \\
& \sigma_{x}^{i}=\left(\Delta_{i} / E_{i}\right) S_{z}^{i}+\left(\epsilon_{i} / E_{i}\right) S_{x}^{i},
\end{aligned}
$$

where $S_{z}^{i}=\left|0_{i}\right\rangle\left\langle 0_{i}|-| 1_{i}\right\rangle\left\langle 1_{i}\right|$, such an interaction is generated by the

$$
-\frac{\Delta_{i} \Delta_{j}}{4 E_{i} E_{j}} J_{i j} S_{x}^{i} S_{x}^{j}
$$

part of (15). The eigenstates $|1\rangle \equiv\left|0_{i}, 0_{j}\right\rangle,|2\rangle \equiv\left|0_{i}, 1_{j}\right\rangle$, $|3\rangle \equiv\left|1_{i}, 0_{j}\right\rangle,|4\rangle \equiv\left|1_{i}, 1_{j}\right\rangle$ of the Hamiltonian $H_{0, i j}=$ -(1/2) $\left(E_{i} S_{z}^{i}+E_{j} S_{z}^{j}\right)$, (cf. Fig. 2), become mixed due to the interaction term (23). In the up-down subspace spanned by $\left|0_{i}, 1_{j}\right\rangle=\left|0_{i}\right\rangle \otimes\left|1_{j}\right\rangle$ and $\left|1_{i}, 0_{j}\right\rangle=\left|1_{i}\right\rangle \otimes\left|0_{j}\right\rangle$ this pair coupling can effectively be described by a TLSHamiltonian of the type (11) with $\sigma_{z}=\left|0_{i}, 1_{j}\right\rangle\left\langle 0_{i}, 1_{j}\right|-$ $\left|1_{i}, 0_{j}\right\rangle\left\langle 1_{i}, 0_{j}\left|, \sigma_{x}=\right| 0_{i}, 1_{j}\right\rangle\left\langle 1_{i}, 0_{j}|+| 1_{i}, 0_{j}\right\rangle\left\langle 0_{i}, 1_{j}\right|$, and pair asymmetry energy, pair tunneling frequency, and pair level splitting

$$
\begin{aligned}
\epsilon_{p} & =E_{i}-E_{j}, \\
\Delta_{p} & =J_{i j} \Delta_{i} \Delta_{j} / 2 E_{i} E_{j}, \\
E_{p} & =\sqrt{\Delta_{p}^{2}+\epsilon_{p}^{2}} .
\end{aligned}
$$

The eigenstates for the coherently coupled pair then read

$$
\begin{aligned}
& |+\rangle=\sqrt{\frac{1+\epsilon_{p} / E_{p}}{2}}\left|0_{i}, 1_{j}\right\rangle+\sqrt{\frac{1-\epsilon_{p} / E_{p}}{2}}\left|1_{i}, 0_{j}\right\rangle, \\
& |-\rangle=\sqrt{\frac{1+\epsilon_{p} / E_{p}}{2}}\left|1_{i}, 0_{j}\right\rangle-\sqrt{\frac{1-\epsilon_{p} / E_{p}}{2}}\left|0_{i}, 1_{j}\right\rangle .
\end{aligned}
$$

Clearly, the more asymmetric the pair is, i.e., the larger the energy offset $E_{i}-E_{j}$ is, the more localized at one TLS is the pair excitation.

Based on the distribution (2) of the single TLS tunneling parameters and a uniform spatial distribution of the single TLS in the glass, Burin and Kagan 16 derived the following distribution function for the parameters of coherently coupled pairs

$$
P^{(2)}\left(\epsilon_{p}, \Delta_{p}\right)=\frac{\pi^{3}}{12}\left(P_{0} k_{B} T\right)\left(P_{0} U_{0}\right) \frac{1}{\Delta_{p}^{2}} \Theta\left(\Delta_{p}-\Delta_{p, \min }\right),
$$

where $\Theta(x)$, the unit step function, is included to emphasize that the distribution has a cutoff at small $\Delta_{p}$. Note that the density of states of the pairs is linearly temperature dependent. Coherent coupling between pairs is destroyed if there is spontaneous decay during the updown transition. For primary TLS with $E \approx 2 k_{B} T$ this occurs with a rate (4), which guided Burin and Kagan to estimate the lower bound (for secondary TLS formed from symmetric primary TLS)

$$
\Delta_{p, \min }=\hbar \alpha T^{3} .
$$

Comparing (27) with (7) yields

$$
A(T)=\left(\pi^{3} / 12\right)\left(P_{0} U_{0}\right) k_{B} T .
$$

This looks encouraging; however, after a closer examination there are several inconsistencies. First, Maier et al.12 could fit their data with a temperature independent parameter A. Burin and Kagan's theory gives $A \propto T$. The $10 \%$ variation, which Maier et al.12 found between the 1 and $0.5 \mathrm{~K}$ data, is too weak to account for the linear temperature dependence of the theory. Second, putting in numbers, we find for PMMA at $1 \mathrm{~K}$ : $A(1 \mathrm{~K}) / k_{B} \approx 10^{-4} \mathrm{~K}$ and $\Delta_{p, \min } / k_{B} \approx 400 \mathrm{mK}$, which is inconsistent with the experimental values (13), (14) by several orders of magnitude. Indeed, at $1 \mathrm{~K}$, TLS with $\Delta_{\min } / k_{B} \sim 0.4 \mathrm{~K}$ can never be responsible for spectral diffusion on the time scale between $10^{3}$ and $10^{6} \mathrm{~s}$. However, it is possible that very asymmetric primary TLS are responsible for long time spectral diffusion. For very asymmetric TLS, the estimate of Burin and Kagan (28) would be significantly reduced. Hence, though Burin and Kagan's theory predicts the measured time-dependence quite accurately, there arise severe inconsistencies in orders of magnitude and the temperature dependence upon applying their theory at $1 \mathrm{~K}$. It should be mentioned that Burin and Kagan considered only the millikelvin regime, which avoids all these problems.

\section{THE KASSNER-SILBEY APPROACH FOR PRIMARY AND SECONDARY TLS}

Though the picture developed by Burin and Kagan is very appealing, it explains on qualitatively the holeburning data of Maier et al.11,22. The question arises whether a microscopic calculation can yield testable predictions based on the interacting TLS scheme of Burin and Kagan. We carry out calculations based on strong 
coupling of TLS to phonons with deformation potential $\gamma \approx 1 \mathrm{eV}$. This allows for a shifting of the rate distribution towards longer times and indeed brings the "calculated" value of $A$ (see (29)) into closer agreement with experiment. Furthermore, the inclusion of strong coupling effects alone gives a reasonable fit to the experiment of Maier et al. for intermediate times (cf. Fig. 3(a)).

Let us start with the usual spin-boson Hamiltonian for an ensemble of TLS interacting with phonons via a strain field. In the TLS-eigenbasis the Hamiltonian reads

$$
\begin{aligned}
H= & -\frac{1}{2} \sum_{i} E_{i} S_{z}^{i}+\sum_{i, q}\left(\bar{u}_{i} S_{z}^{i}-u_{i} S_{x}^{i}\right) c_{q}^{i}\left(b_{q}+b_{-q}^{\dagger}\right) \\
& +\sum_{q} \hbar \omega_{q} b_{q}^{\dagger} b_{q}
\end{aligned}
$$

with

$$
c_{q}^{i}=\mathrm{i} \gamma q\left(\frac{1}{2 \varrho V \omega_{q}}\right)^{1 / 2} e^{\mathrm{iqr}_{i}}
$$

and

$$
u_{i}=\Delta_{i} / E_{i}, \quad \bar{u}_{i}=\epsilon_{i} / E_{i}
$$

According to this, the TLS become dressed with clouds of virtual phonons. As a result the coupling between the TLS will also be changed. The assumption traditionally made is that the dressed entities can be considered as weakly interacting. Hence, first order perturbation theory in the dressed states might be sufficient at low temperatures.

Based on this picture, Kassner and Silbey 17 derived a $S_{z}^{i} S_{z}^{j}$-interaction between TLS and a reduction of relaxation rates for asymmetric TLS. Compared to Eq. (1), they found well below the Debye-temperature of the glass, $T \ll \Theta_{\mathrm{D}}$,

$$
R=\left(\frac{\Delta}{E}\right)^{2} e^{-G(\epsilon / E)^{2}} R_{\max }
$$

where [cf. Eq. (6)]

$$
G=\left(\hbar / 8 \pi k_{B}\right) \alpha \Theta_{D}^{2} .
$$

It is a peculiar feature of their approach that symmetric TLS have no reduced rates, i.e., $R_{\max }$ is still given by Eq. (5), and have zero interaction. It is this very fact which significantly changes the distribution function $P^{(1)}(E, r)$ of single TLS energies $E$ and dimensionless relaxation rates $r=R / R_{\max }$ compared to the STM-result (cf. first term in Eq. (8)). According to (33)

$$
r=\left(\frac{\Delta}{E}\right)^{2} e^{-G(\epsilon / E)^{2}}
$$

(compared to the STM-result $\left.r=(\Delta / E)^{2}\right)$. With this, Kassner and Silbey derived the new distribution

$$
P^{(1)}(E, r)=\frac{P_{0}}{2 r \bar{u}(r)\left\{1+G\left[1-\bar{u}^{2}(r)\right]\right\}},
$$

where $\bar{u}(r)=\epsilon / E$ is the inverse function of

$$
r(\bar{u})=\left(1-\bar{u}^{2}\right) e^{-G \bar{u}^{2}} .
$$

The result is a stretching of the distribution in $r$ towards smaller values, i.e., $P^{(1)}(r)$ has an extended tail for such rates. The flaws and merits of the Kassner-Silbey way of handling strong-TLS-phonon-coupling effects have been discussed in Ref. 17. An important point is that a $S_{x}^{i} S_{x}^{j}$ interaction between TLS, as used in Burin and Kagan's approach, cannot be derived from this approach.

In Appendix A we have generalized Kassner and Silbey's approach to include coherent coupling between pairs. Our procedure has been as follows. First, we have eliminated the diagonal $\left(S_{z}^{i}\right)$ and off-diagonal $\left(S_{x}^{i}\right)$ coupling in the Hamiltonian (30) by two separate unitary transformations (A.1) and A.6. Second, instead of continuing with the full transformed Hamiltonian, we have projected out the one-phonon coupling part in the dressed state basis. This has been achieved by expanding the generated phonon shift operators around their mean value up to the first leading term in $c_{q}^{i}$. This generates one-phonon transition matrix elements in the four-level system of the pair and, more importantly, Debye-Waller factors which renormalize Burin and Kagan's coherent coupling term 23 as

$$
-J_{i j} \Delta_{i} e^{-G\left(\epsilon_{i} / E_{i}\right)^{2} / 2} \Delta_{j} e^{-G\left(\epsilon_{j} / E_{j}\right)^{2} / 2} /\left(4 E_{i} E_{j}\right) S_{x}^{i} S_{x}^{j}
$$

Here,

$$
\frac{1}{4} J_{i j}=\sum_{q} \frac{c_{q}^{i} c_{-q}^{j}}{\hbar \omega_{q}}
$$

which is equivalent to (16)-(20). The pair asymmetry energy and tunneling amplitude read

$$
\begin{aligned}
\epsilon_{p} & =E_{i} e^{-W_{i} G u_{i}^{2} / 2}-E_{j} e^{-W_{j} G u_{j}^{2} / 2}, \\
\Delta_{p} & =J_{i j} \Delta_{i} e^{-G \bar{u}_{i}^{2} / 2} \Delta_{j} e^{-G \bar{u}_{j}^{2} / 2} / 2 E_{i} E_{j},
\end{aligned}
$$

with $W_{i}=e^{-G \bar{u}_{i}^{2} / 2}$. One should note that for $G \gg 1$ the Debye-Waller factor $e^{-W_{i} u_{i}^{2} / 2}$ is practically unity except for nearly symmetric TLS. Since we will be mainly interested in strongly asymmetric TLS, we will always use this simplification hereafter. In Appendix B we calculate the pair parameter distribution function. The result is:

$$
P^{(2)}\left(\epsilon_{p}, \Delta_{p}\right)=P^{(2)}\left(\Delta_{p}\right) P^{(2)}\left(\epsilon_{p}\right)
$$

with

$$
P^{(2)}\left(\Delta_{p}\right)=\frac{P_{0}}{\Delta_{p}^{2}} e^{-G / 2} I_{0}^{2}(G / 4) \Theta\left(\Delta_{p}-\Delta_{p, \min }\right)
$$


where $I_{0}(z)$ is a modified Bessel function, and

$$
P^{(2)}\left(\epsilon_{p}\right)=f\left(\epsilon_{p}\right)+f\left(-\epsilon_{p}\right),
$$

with

$$
\begin{aligned}
f\left(\epsilon_{p}\right) & =\frac{A(T)}{1-e^{-\beta \epsilon_{p}}} \Theta\left(E_{\max }-\epsilon_{p}\right) \times \\
& \times\left\{\log \left(\frac{2}{1+e^{-\beta \epsilon_{p}}}\right)-\log \left(\frac{1+e^{-\beta E_{\max }}}{1+e^{-\beta\left(\epsilon_{p}+E_{\max }\right)}}\right)\right\} .
\end{aligned}
$$

The parameters $E_{\max }, \Delta_{p, \min }$ are cutoffs which are set by the requirement of stability for the coupled pairs. $E_{\max }$ is a cutoff in the energy of the primary TLS, which, as we will see below, is generally a function of the temperature. If $E_{\max } \gg k_{B} T, P^{(2)}\left(\epsilon_{p}\right)$ is the sum of a constant and a bell shaped curve centered around $\epsilon_{p}=0$ with a width of $O\left(k_{B} T\right)$. It can be approximated by

$$
P^{(2)}\left(\epsilon_{p}\right) \approx A(T)\left[\log 2+(1-\log 2) \exp \left(-\beta^{2} \epsilon_{p}^{2} / 9\right)\right]
$$

for all practical purposes. If $E_{\max } \ll k_{B} T$, the distribution $P^{(2)}\left(\epsilon_{p}\right)$ becomes independent of $\epsilon_{p}$,

$$
P^{(2)}\left(\epsilon_{p}\right) \approx A(T) E_{\max }(T) / 4 k_{B} T .
$$

In the limit $E_{\max } \gg k_{B} T$ and $G, \epsilon_{p} / k_{B} T \ll 1$, we find Burin and Kagan's result (27). This confirms that their model is valid at ultralow temperatures for nearly symmetric TLS with weak coupling to phonons. In the limit $G \gg 1$, the asymptotic expansion of the modified Bessel function, $I_{0}(z) \approx e^{z} / \sqrt{2 \pi z},(z \gg 1)$, provides a renormalization of $P_{0}$ by a factor $2 /(\pi G)$.

\section{STABILITY ANALYSIS}

To determine $E_{\max }$ and $\Delta_{p \text {,min }}$, we first neglect the level broadening effect of the phonons. Then, $E_{\max }$ scales with the glass transition temperature, and $\Delta_{p \text {,min }}$ is set by the concentration $N_{p}$ of pairs in the glass. To determine $N_{p}$, we start with estimating the probability for 2 TLS separated by a distance $\mathbf{r}$ to form a pair to $P(\mathbf{r}) \approx P_{0} U_{0} /(8 \pi / 3)|\mathbf{r}|^{3}$. Then the concentration of pairs in a shell $[V-V / 2, V+V / 2]$ is given by $N_{p} \approx \int_{V / 2}^{3 V / 2} n P(\mathbf{r}) d \mathbf{r}$, where $n=P_{0} k_{B} T$ is the number of thermal TLS. This yields

$$
N_{p}=(1 / 2)\left(P_{0} T\right)\left(P_{0} U_{0}\right) \log 3,
$$

which in three dimensions is independent of the volume $V$ due to the $|\mathbf{r}|^{-3}$-law of interaction. The maximum volume $V_{*}=(4 \pi / 3)\left|\mathbf{r}_{*}\right|^{3}$ each of these pairs can occupy is given by $1 / N_{p}$ which determines the minimum interaction energy $J_{\min }=U_{0} /\left|\mathbf{r}_{*}\right|^{3}$. This provides according to Eq. (41)

$$
\Delta_{p, \min }=\frac{1}{2} J_{\min }=\frac{\pi}{3}\left(P_{0} U_{0}\right)^{2} k_{B} T
$$

as a reasonable estimation of the lower bound. Indeed, for PMMA, Eq. (49) provides $\Delta_{p, \min } / k_{B}=10^{-8} \mathrm{~K}$ which, at $1 \mathrm{~K}$, corresponds to a maximum relaxation time $\tau_{\max }=10^{6}$ to $10^{7} \mathrm{~s}$.

Let us now include decoherence effects by phonons. We will not provide a full discussion of the relaxation dynamics in the four-level system of the pair, but instead try to argue more physically. After two unitary transformations (A.1) and (A.6), one finds the Hamiltonian (A.11) as is pointed out in Appendix A. Here, only the term $\propto S_{x} \otimes S_{y}$ allows relaxation within the up-down subspace $\{|0,1\rangle,|1,0\rangle\}$. The relaxation mechanism is a flip-flop process linked with the emission or absorption of a phonon. The relaxation rate scales with $\left(\Delta_{p} / E_{p}\right)^{2}$ :

$$
R^{(p)}=r_{p} R_{\max }^{(p)},
$$

where

$$
\begin{aligned}
r_{p} & =\left(\frac{\Delta_{p}}{E_{p}}\right)^{2} \\
R_{\max }^{(p)} & \approx \alpha T^{3} x_{p}^{3} \operatorname{coth} x_{p}
\end{aligned}
$$

and $x_{p}=E_{p} / 2 k_{B} T$. We used that $\alpha_{p} \approx \alpha$, as discussed in Appendix A. Note that there is no Debye-Waller factor of the Kassner-Silbey type because of the coupling of the phonons to $\sigma_{y}^{p}$ instead of to $\sigma_{z}^{p}$. The coherent coupling is destroyed by spontaneous decay during the up-down transition of primary TLS constituting a pair: for instance $|0,1\rangle,|1,0\rangle \rightarrow|0,0\rangle$. Based on this argument, Burin and Kagan estimated $\Delta_{p, \min }=\hbar \alpha T^{3}$, where $\alpha T^{3}$ is the decay rate of symmetric, primary TLS with $E=2 k_{B} T$. Clearly, on a the time scale explored in the experiment of Maier et al., strongly asymmetric TLS dominate the hole width instead of symmetric ones. Note that in constructing $P^{(2)}\left(\epsilon_{p}, \Delta_{p}\right)$ (Eq. (42)), we integrated over values of the energy splittings of the primary TLS less than $E_{\max }(T)$, which have the correct initial population factors to insure the creation of stable pair excitations. We now investigate which primary TLS are able to guarantee stability of secondary TLS at $1 \mathrm{~K}$, i.e., we ask whether the limit $E_{\max }(T)>k_{B} T$ or $<k_{B} T$ applies. We first study if secondary TLS may be formed from primary TLS with thermal splitting, $E \sim O\left(k_{B} T\right)$, at $1 \mathrm{~K}$. We need to satisfy three conditions. First, we require

$$
E_{p} / \hbar \geq R\left(E=2 k_{B} T\right),
$$

where $R\left(E=2 k_{B} T\right) \equiv r \alpha T^{3} \leq 1 / t$ is the relaxation rate of a primary thermal TLS which has not yet decayed at $t$, i.e., for which (cf. Eq. (33))

$$
1 / r \geq \alpha T^{3} t \approx 10^{13}-10^{16}
$$

with $t=10^{3}-10^{6} \mathrm{~s}$. These two requirements guarantee that the secondary TLS is coherent on the time 
scale where deviations from logarithmic spectral diffusion is seen. Furthermore, if secondary TLS are responsible for the spectral line broadening after $10^{3} \mathrm{~s}$, the pair rate must satisfy $R^{(p)} t=1$. This provides the relation $\left(\Delta_{p} / E_{p}\right)^{2}=1 / \alpha T^{3} t x_{p}^{3} \operatorname{coth} x_{p}$. Using this relation, multiplying Eq. (53) by $1 / E_{p}$ and squaring it, one can now easily check that Eq. (54) always implies Eq. (53).

With respect to the stability of secondary TLS at $1 \mathrm{~K}$, we conclude: First, from the condition (54) we deduce the criterion $r \ll 1$, i.e, the primary TLS must be very asymmetric. Second, upon replacing $\Delta_{p}=J r$ [cf. Eq. (41)] and noting that $x_{p} / \sqrt{x_{p}^{3} \operatorname{coth} x_{p}} \approx 1$ for $0 \leq x_{p} \leq 1$, we find the criterion

$$
\frac{J}{2 k_{B} T}>\sqrt{\alpha T^{3} t} \approx 10^{6}-10^{8}
$$

Hence, according to $J=U_{0} / d^{3}$ and $U_{0} \approx 10^{4} \mathrm{~K} \AA^{3}$, the relevant TLS are separated by a distance $d \lesssim 1 \AA$. Note that these estimates are highly approximate. In particular, we have approximated the rate at which coherence in a secondary TLS is destroyed by the relaxation rate of a primary TLS, without consideration of the true rates that govern the coupled four level system. However, we may still conclude that at $1 \mathrm{~K}$, it is unlikely that primary TLS with thermal splitting, $E \sim O\left(k_{B} T\right)$, can form pairs, unless they sit extremely close to each other. This fact suggests a natural cutoff, $E_{\max }(T)$, in the energy splittings of the primary TLS that comprise the secondary TLS existing at $1 \mathrm{~K}$. This cutoff is motivated by the above stability criteria, and can change the temperature dependence of $P^{(2)}\left(\epsilon_{p}\right)$ in Eq. (44) and of the hole width in general. We will return to this point in the next section. According to this, the distribution $P^{(2)}\left(E_{p}, r_{p}\right)$ reads

$$
P^{(2)}\left(E_{p}, r_{p}\right)=P^{(2)}\left(r_{p}\right) P^{(2)}\left(E_{p}, \bar{r}_{p}\right)
$$

where

$$
P^{(2)}\left(r_{p}\right)=\frac{P_{0} e^{-G / 2} I_{0}^{2}(G / 4)}{2 r_{p}^{3 / 2} \sqrt{1-r_{p}}} \Theta\left(r_{p}-r_{p, \min }\right)
$$

and

$$
\begin{aligned}
& P^{(2)}\left(E_{p}, \bar{r}_{p}\right)=\frac{A(T)}{\left|E_{p}\right|\left(1-e^{\left.-\bar{r}_{p} \beta E_{p}\right)}\right.} \times \\
& \times\left\{\log \left(\frac{2}{1+e^{-\beta \bar{r}_{p} E_{p}}}\right)-\log \left(\frac{1+e^{-\beta E_{\max }}}{1+e^{-\beta\left(\bar{r}_{p} E_{p}+E_{\max }\right)}}\right)\right\} \\
& +\left(E_{p} \rightarrow-E_{p}\right),
\end{aligned}
$$

with $0<E_{p} \leq E_{\max }(T), \bar{r}_{p}=\sqrt{1-r}$, and $A(T)$ given by Eq. (29).

\section{COMPARISON WITH EXPERIMENT}

We now calculate the broadening of a spectral hole in the inhomogeneous line due to spectral diffusion induced by siagle TLS and pairs. According to the standard theory 11 , the experimentally observed line broadening, $\Delta \Gamma(t) \equiv \Gamma(t)-\Gamma\left(t_{0}\right)$, can be written as

$$
\begin{aligned}
& \Delta \Gamma(t)=\frac{\pi^{2}}{3 \hbar}\langle C\rangle \int_{0}^{\infty} d E \operatorname{sech}^{2} \frac{E}{2 k_{B} T} \times \\
& \times \int_{0}^{1} d r\left(\bar{u}(r) P^{(1)}(E, r)+\sqrt{1-r} P^{(2)}(E, r)\right) \\
& \times\left(e^{-r R_{\max } t_{0}}-e^{-r R_{\max } t}\right)
\end{aligned}
$$

where $P^{(1)}(E, r)$ and $P^{(2)}(E, r)$ is given by (36) and (56), respectively. To calculate these integrals, we replace the last factor by the step function, which restricts the $r$ integration to the interval $\left[1 / R_{\max } t, 1 / R_{\max } t_{0}\right]$. This gives

$$
\Delta \Gamma(t)=\Delta \Gamma^{(1)}(t)+\Delta \Gamma^{(2)}(t)
$$

where

$$
\Delta \Gamma^{(1)}(t)=\frac{\pi^{2}}{3 \hbar}\langle C\rangle P_{0} k_{B} T \log \frac{1-\bar{u}^{2}\left(1 / \alpha T^{3} t_{0}\right)}{1-\bar{u}^{2}\left(1 / \alpha T^{3} t\right)}
$$

is the contribution of the primary TLS to the spectral hole width. To calculate the pair contribution $\Delta \Gamma^{(2)}(t)$, we consider the limits $E_{\max }(T) \gg k_{B} T$ and $\ll k_{B} T$, separately.

\section{A. The limit $E_{\max }(T) \gg k_{B} T$}

Remember, in this limit coherently coupled pairs can be built by thermal primary TLS. We find from Eq. (60)

$$
\begin{aligned}
\Delta \Gamma^{(2)}(t) & =\frac{\pi^{2}}{3 \hbar} e^{-G / 2} I_{0}^{2}(G / 4)\langle C\rangle P_{0} \times \\
& \times A(T) \sqrt{\alpha T^{3}}\left(\sqrt{t}-\sqrt{t_{0}}\right) .
\end{aligned}
$$

Putting Eqs. (61)-63) together provides an equation which is very similar to the result (11). However, this equation is based on the microscopic picture of phononmediated TLS-TLS interaction. There are two regimes where the function $\bar{u}\left(1 / \alpha T^{3} t\right)$ can be determined analytically: (i) short-time limit: $\alpha T^{3} t \ll e^{G}$ which gives $\bar{u}^{2}\left(1 / \alpha T^{3} t\right) \approx \frac{1}{1+G} \log \left(\alpha T^{3} t\right)$; (ii) longtime limit: $\alpha T^{3} t \gg e^{G}$ which gives $\bar{u}^{2}\left(1 / \alpha T^{3} t\right) \approx 1-e^{G} / \alpha T^{3} t$. In Ref. 12(b), Maier and Haarer have fit their data with only the first term in (61). By numerical inversion of Eq. (37), they could find good agreement on intermediate time scales up to $200-300 \mathrm{~min}$. We have illustrated this in Fig. 3(a). The values for the fit parameters $G$ and $P_{0}\langle C\rangle$ are 32 and $6 \times 10^{-5}$, respectively. With this value for $G$, the crossover from the short- to the longtime behavior happens after ca. $150 \mathrm{~min}$; Eqs. (12) und (34) yield a Debye-temperature of $\Theta_{D}=108 \mathrm{~K}$, which is in reasonable agreement with literature data for PMMA. It 
is in part due to the success of the Kassner-Silbey theory at intermediate times that we have adopted the strong coupling approach as our dynamical starting point. For times larger than 300 min, Maier and Haarer attributed the deviations of the theory from the experimental data the contribution of interacting TLS. If this is true, we should find agreement between theory and experiment when including the second term in (61). In Fig. 3(b) we have plotted $\Delta \Gamma(t)$, Eqs. (61)-(63), together with the experimental data of Ref. 12 for $1 \mathrm{~K}$ (upper curve) and $0.5 \mathrm{~K}$ (lower curve). We used the same value for $G$ as in the previous plot, and have optimized the TLS-TLS coupling parameter, $P_{0} U_{0}$, and the TLS-chromophore coupling parameter, $P_{0}\langle C\rangle$, to find best agreement for the $0.5 \mathrm{~K}$ data. The result is $P_{0} U_{0}=2.5 \times 10^{-6}$ and $P_{0}\langle C\rangle=4 \times 10^{-5}$. The upper curve shows the prediction of the theory for the $1 \mathrm{~K}$ data. The parameter value for $P_{0} U_{0}$, which fits the $0.5 \mathrm{~K}$ data is by a factor $25-$ 35 smaller than the literature value. 18 However, we see that a superposition of a $\log t$ - and a $t^{0.5}$-term can be interpreted as an effective $t^{0.38}$-power law on the experimental time scale as seen by Maier et al. Hence, under the assumption that primary TLS with thermal energy splitting can form stable secondary TLS at $1 \mathrm{~K}$, we find $\Delta \Gamma^{(2)} \propto T^{5 / 2}$, giving a temperature dependence that is too strong compared with the experimental observation which indicates $\Delta \Gamma^{(2)} \propto T^{3 / 2}$, at least in fitting the data at the two temperatures, $0.5 \mathrm{~K}$ and $1 \mathrm{~K}$. This can clearly be seen by the prediction of the theory for the data in Fig. 3(b).

\section{B. The limit $E_{\max }(T) \ll k_{B} T$}

We have noted in the previous section that it is very unlikely that thermal primary TLS can form stable pairs at $1 \mathrm{~K}$. If we impose a minimum separation distance between primary TLS (say $5 \AA$ ), then a natural energy cutoff enters due to the stability requirements outlined in the previous section. If we assume that this cutoff $E_{\max }$ satisfies the condition

$$
E_{\max }(T) \ll k_{B} T,
$$

then we can estimate $E_{\max }(T)$ from the conditions considered in Sec. IV. Specifically, from $R t \sim 1, R^{(p)} t \sim 1$, $x_{p} / \sqrt{x_{p}^{3} \operatorname{coth} x_{p}} \sim 1$, and $r=\Delta_{p} / J$,

$$
\left(\frac{E_{\max }(T)}{2 k_{B} T}\right)^{3} \operatorname{coth}\left(\frac{E_{\max }(T)}{2 k_{B} T}\right) \sim \frac{J_{\max }}{2 k_{B} T \sqrt{\alpha T^{3} t}}
$$

where $J_{\max }=U_{0} / d_{\min }^{3}$. This shows that $E_{\max }(T) \sim$ $T^{-1 / 4}$, which significantly alters the temperature dependence of the hole width. If the above condition is met, then

$$
P^{(2)}\left(E_{p}\right) \approx A(T) E_{\max }(T) / 4 k_{B} T \sim T^{-1 / 4}
$$

and

$$
\begin{aligned}
\Delta \Gamma^{(2)}(t) & \approx \frac{\pi^{2}}{6 \hbar} e^{-G / 2} I_{0}(G / 4)\langle C\rangle P_{0}\left(\sqrt{t}-\sqrt{t_{0}}\right) \times \\
& \times \sqrt{\alpha T^{3}} A(T)\left(\frac{E_{\max }(T)}{2 k_{B} T}\right)^{2}
\end{aligned}
$$

In the regime where $E_{\max }(T) \ll k_{B} T$, this expression is essentially temperature independent. Thus, the stability requirements imply an interesting thermal breakup of the secondary TLS.

\section{DISCUSSION}

For very low temperatures $\left(E_{\max }(T) \gg k_{B} T\right)$, the majority of asymmetric TLS are stable even if constructed from asymmetric primary TLS that have energy splittings on the order of $k_{B} T$, leading to the strong temperature dependence depicted in Fig. 3(b). We have argued that at higher temperatures, a crossover should occur where the temperature dependence should become weaker, as secondary TLS become less stable. Therefore, it is qualitatively consistent with this picture that the observed temperature dependence at $\sim 1 \mathrm{~K}$ is weaker than that shown in Fig. 3(b). Such arguments require further "slowing down" of the temperaturedependence. This is indeed seen experimentally at $2 \mathrm{~K} .20$ Hannig et al. are able to fit their data with essentially the same value of $A$ at $0.5 \mathrm{~K}$ and $1 \mathrm{~K}$, and a value of $0.3 \times A(T=0.5 \mathrm{~K})$ at $2 \mathrm{~K}$. Eventually, the entire TLS picture should break down at some temperature in the range 1-10 K. A clear test of the validity of this picture would be to see if the stronger temperature dependence emerges at lower temperatures. Such experiments are difficult to conduct at ultralow temperatures, due to slow equilibration effects.

We may also note that the discrepancy between the "derived" and literature values of $P_{0} U_{0}$ can be explained by the thermal breakup of secondary TLS demanded by stability criteria. As the temperature is raised, the primary TLS must lie closer together to form a stable secondary TLS at long times. The probability of finding two neighboring primary TLS a very close distance apart is small, which effectively decreases the "derived" value of $P_{0} U_{0}$. Note that there is also a reduction in $\Delta \Gamma^{(2)}(t)$ due to the factor $\left(E_{\max } / 2 k_{B} T\right)^{2}$ (see Eq. (67)). Thus, at least qualitatively, it is indeed possible that a picture based on coupled TLS can account for all the properties currently observed in longtime spectral diffusion experiments.

So far we have shown the pros and cons of applying the idea of coupled pairs to hole-burning experiments on ultralong time scales at $1 \mathrm{~K}$. Here we propose an interesting experiment which, although difficult to perform, would provide a conclusive test of the coupled TLS hypothesis. A crucial observation is that the exponent of the power law depends on the dimensionality of the 
glassy probe. For example, the exponent would be systematically smaller if the primary TLS were confined to (quasi)-two dimensions, while the interaction between them still varies as $1 /|\mathbf{r}|^{3}$. This would result in a distribution function $P\left(\Delta_{p}\right) \propto \Delta_{p}^{5 / 3}$ (from $P\left(\Delta_{p}\right) \propto P(J)=$ $P(|\mathbf{r}|)|\mathbf{r}|(|d| \mathbf{r}|/ d J|)$ and $J \propto|\mathbf{r}|^{-3}, P(|\mathbf{r}|)=$ constant $)$ and therefore in a $t^{1 / 3}$-power law. The experiment we suggest has already been performed, albeit not not for the purpose that we discuss here and not on a time scale up to $10^{6} \mathrm{~s}$. The hole-burning experiment by Orrit et al.21 on an ionic dye in a Langmuir-Blodgett monolayer lying on a three-dimensional substrate is an experiment of the type we proposed above - the TLS dynamics is restricted to two dimensions whereas sound waves and strain are not affected by the interface between the amorphous layer and the bulk. Indeed, Orrit et al 21 have observed spectraldiffusion which could be explained by Pack and Fayer22 using the standard tunneling model. From this perspective it seems promising to extend Orrit's experiment to longer times and look whether a power law weaker than the three-dimensional result $t^{0.5}$ could be observed.

There are, however, reasons to be skeptical of the picture we have outlined. A number of approximations have been invoked that render only a semiquantitative description of the experimental results. These approximations include the reduction of the primary TLS to an effective secondary TLS, and the use of relaxation rates for uncoupled TLS to discuss decoherence effects for coupled secondary TLS. These approximations, especially the first one, may not be valid at temperatures near $1 \mathrm{~K}$. Clearly, one has to think about alternative explanations. A simple idea would be to attribute the deviation from the standard $\log t$-behavior to a nonequilibrium state of those TLS which relaxes on these long timescales. Indeed, recent experiments by Friedrich et al.23 have proven that spectral diffusion in glasses under nonequilibrium conditions results in a nonlogarithmic time evolution of the hole width. However, the data in Maier's experiment were obtained after letting the sample relax at the measurement temperature for a longer period than the later data recording period. Hence, one expects that all relaxation processes shorter than this waiting time occur under equilibrium conditions.

It is interesting to compare the glass results with equivalent experiments on proteins. Hole-burning experiments on proteins show almost no hole broadening up to three hours followed by a nonlogarithmic hole broadening regime.24 A ney type of temperature-cycle holeburning experiment 25 leads to the conclusion that the excess broadening of the hole in the protein cannot be interpreted in the framework of the STM. Hence, one might speculate that in both glasses and proteins, the interaction of TLS becomes important at long time scales. An alternative conclusion avoids the notion of interacting TLS altogether. Perhaps the energy landscape of glasses is not too different from that of proteins, and also shows features of organization at high barriers. Recall that the experiments of Maier et al. were carried out in PMMA, which is a polymer glass. Polymer glasses may be expected to have conformations similar to proteins. For example, such "conformational" dynamics may involve side chain motions. The physical picture is that the energy landscape comprises high barriers in addition to constantly distributed lower barriers within each of its basins. The algebraic behavior then results from tunneling through those high barriers, which have to be distributed around a "typical" value $V_{0}$. This value has to be sufficiently high in order that the onset of the algebraic behavior occurs only after $3 \mathrm{~h}$. We give some details on these ideas in Appendix C; more can be found in Ref. 26. The model predicts a temperature and timedependence of the hole width with an exponent which is slightly weaker than $T^{3 / 2}$ and $t^{1 / 2}$, respectively, and a slowly decreasing function of $T$ and $t$. We note that if indeed specific polymer dynamics (like side chain motion) are responsible for deviations from logarithmic spectral diffusion, perhaps a deuterated sample may show different hole broadening behavior.

One is tempted to speculate that these "non-STM like" high barriers arise from the presence of the chromophore in the glassy host27, because they have not been $\mathrm{h}$ in sound attenuation experiments up to 100 K.2829 Note however, that these experiments were not performed on polymer glasses, and that sound attenuation experiments on PMMA and PS indeed show an additional peak above ca. 50 K. 00 Interestingly, doping a network wass with $\mathrm{OH}$-impurities leads to the same observation 29 , which conceivably supports the importants of side chain motion in polymer glasses. Furthermore, in contrast to the glass, the protein probe is not doped by a dye. Instead part of the protein is chemically changed in order to serve as a chromophore. Thus, it seems that such deviations from standard spectral diffusion behavior are not due to the inclusion of the chromophore into the sample.

One should also note nonlogarithmic line broadening is the typical case because a logarithmic time dependence occurs only in case of a $1 / \Delta^{n}$-distribution for the singular case $n=1$. Without invoking the physical reasons for deviations from the standard $1 / \Delta$ distribution function introduced by Anderson, Halperin and Varma, and of Phillips, we may say that for small values of $\Delta$ (corresponding to long times), the distribution of barriers is not really flat, but instead a smoothly varying function of the parameter $\lambda$ (see Appendix C). That the distribution of barriers in such a model turns out to be a log-normal distribution shows a striking similarity to general systems exhibiting $1 / f^{\alpha}$ noise 31

\section{CONCLUSIONS}

In this paper we have analyzed the consistency of the conjecture that coupled pairs of TLS dominate spectral 
diffusion on ultralong time scales. Because the pairs distribution $P^{(2)}\left(\epsilon_{p}, \Delta_{p}\right)$ is correlated with the distribution of the primary "STM-like" TLS, we have in a sense pushed the STM as far as possible by looking at these new low energy excitations. We believe that this is an important step, which has to be done before trying to find another extension of the STM for every new experiment. The questions was whether they also apply to the $1 \mathrm{~K}$ regime, and, in particular, whether they can provide an explanation of the longtime hole-burning experiment of Maier et al. We find that a picture based on the idea of interacting tunneling systems seems consistent with the experimental data, although we are unable to fit certain aspects of the experiment, such as the temperature dependence, quantitatively. Also, alternative explanations have been presented. These models are, at the moment, at least as speculative as the scenario of coherently coupled pairs. For this reason, we have discussed some possible experimental tests of the theoretical models we have presented. More theory and experiments have to be done to finally evaluate the role of coupled TLS in glasses, and to understand the origin of nonlogarithmic hole broadening in glasses.

\section{ACKNOWLEDGEMENTS}

This work has been supported in part by the NSF and the Alexander von Humboldt foundation. We would like to thank A. L. Burin, D. Haarer, Yu. Kagan, B. M. Kharlamov, L. S. Levitov, H. Maier, and R. Wunderlich for helpful discussions.

\section{APPENDIX A:}

In this Appendix we generalize Kassner and Silbey's approach to include coherent coupling between pairs. For this goal we first apply the unitary transformation

$U_{1}=\exp \left[\sum_{i, q}\left(2 \bar{u}_{i} c_{q}^{i} / \hbar \omega_{q}\right)\left(b_{q}-b_{-q}^{\dagger}\right) S_{z}^{i} / 2\right] \equiv \prod_{i} e^{\varphi_{i} S_{z}^{i} / 2}$

to the Hamiltonian (30) which eliminates the diagonal coupling of phonons to $S_{z}^{i}$. The transformed Hamiltonian $H \rightarrow U_{1}^{-1} H U_{1}$ reads with $S_{ \pm}^{j}=(1 / 2)\left(S_{x}^{j} \pm \mathrm{i} S_{y}^{j}\right)$ :17

$$
\begin{aligned}
H & =-\frac{1}{2} \sum_{i} E_{i} S_{z}^{i}+\sum_{q} \hbar \omega_{q} b_{q}^{\dagger} b_{q} \\
& -\sum_{i, q} u_{i} c_{q}^{i}\left(b_{q}+b_{-q}^{\dagger}\right)\left(B_{-}^{i} S_{+}^{i}+B_{+}^{i} S_{-}^{i}\right) \\
& -\frac{1}{4} \sum_{i, j} J_{i j}\left(\bar{u}_{i} \bar{u}_{j} S_{z}^{i} S_{z}^{j}-2 u_{i} \bar{u}_{j}\left(B_{-}^{i} S_{+}^{i}+B_{+}^{i} S_{-}^{i}\right) S_{z}^{j}\right),
\end{aligned}
$$

Expanding again around the one-phonon fluctuations around the shifted harmonic oscillator coordinate by replacing $\widetilde{S}_{z}^{j} \approx S_{z}^{j}\left\langle D_{ \pm}^{j}\right\rangle-\mathrm{i} \phi_{j} S_{y}^{j}$ and $\widetilde{S}_{y}^{j} \approx S_{y}^{j}\left\langle D_{ \pm}^{j}\right\rangle+\mathrm{i} \phi_{j} S_{z}^{j}$, where

$$
\left\langle D_{ \pm}^{j}\right\rangle=e^{-G\left(\Delta_{j}\left\langle B_{ \pm}^{j}\right\rangle / E_{j}\right)^{2} / 2}
$$

one finds

$$
\frac{1}{4} J_{i j}=\sum_{q} \frac{c_{q}^{i} c_{-q}^{j}}{\hbar \omega_{q}}
$$

and

$$
B_{ \pm}^{i}=\exp \left[ \pm \sum_{q}\left(2 \bar{u}_{i} c_{q}^{i} / \hbar \omega_{q}\right)\left(b_{q}-b_{-q}^{\dagger}\right)\right] \equiv e^{ \pm \varphi_{i}}
$$

The $B_{ \pm}^{i}$ are the usual phonon shift operators. Instead of proceeding with the full transformed Hamiltonian, we project out the one-phonon fluctuations around the shifted harmonic oscillators coordinates. This is achieved by expanding $B_{ \pm}^{i} \equiv\left\langle B_{ \pm}^{i}\right\rangle+\left(B_{ \pm}^{i}-\left\langle B_{ \pm}^{i}\right\rangle\right)=\left\langle B_{ \pm}^{i}\right\rangle \pm \varphi_{i}$,

$$
\left\langle B_{ \pm}^{i}\right\rangle=e^{-G\left(\epsilon_{i} / E_{i}\right)^{2} / 2}
$$

and neglecting two-phonon terms. Applying Fermi's Golden Rule to the remaining one-phonon term directly yields the rate (33). A coherent coupling between pairs is generated by eliminating this term by a second unitary transformation

$$
\begin{aligned}
U_{2} & =\exp \left[-\sum_{i, q}\left(2 u_{i}\left\langle B_{ \pm}^{i}\right\rangle c_{q}^{i} / \hbar \omega_{q}\right)\left(b_{q}-b_{-q}^{\dagger}\right) S_{x}^{i} / 2\right] \\
& \equiv \prod_{i} e^{-\phi_{i} S_{x}^{i} / 2} .
\end{aligned}
$$

This yields:

$$
\begin{aligned}
H & =-\frac{1}{2} \sum_{i} E_{i} \widetilde{S}_{z}^{i}+\sum_{q} \hbar \omega_{q} b_{q}^{\dagger} b_{q} \\
& -\frac{1}{4} \sum_{i, j} J_{i j}\left\{\bar{u}_{i} \bar{u}_{j} \widetilde{S}_{z}^{i} \widetilde{S}_{z}^{j}+u_{i}\left\langle B_{ \pm}^{i}\right\rangle u_{j}\left\langle B_{ \pm}^{j}\right\rangle S_{x}^{i} S_{x}^{j}\right. \\
& \left.-2 u_{i} \bar{u}_{j}\left(\left\langle B_{ \pm}^{i}\right\rangle S_{x}^{i}-\mathrm{i} \varphi_{i} \widetilde{S}_{y}^{i}\right) \widetilde{S}_{z}^{j}\right\}
\end{aligned}
$$

where

$$
\begin{gathered}
\widetilde{S}_{z}^{j}=S_{z}^{j} \cosh \phi_{j}-\mathrm{i} S_{y}^{j} \sinh \phi_{j} \\
\widetilde{S}_{y}^{j}=S_{y}^{j} \cosh \phi_{j}+\mathrm{i} S_{z}^{j} \sinh \phi_{j} .
\end{gathered}
$$

政

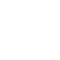




$$
\begin{aligned}
H & =-\frac{1}{2} \sum_{i} E_{i}\left\langle D_{ \pm}^{i}\right\rangle S_{z}^{i}+\sum_{q} \hbar \omega_{q} b_{q}^{\dagger} b_{q}+\frac{\mathrm{i}}{2} \sum_{i} E_{i} \phi_{i} S_{y}^{i} \\
& -\frac{1}{4} \sum_{i, j} J_{i j}\left\{\bar{u}_{i}\left\langle D_{ \pm}^{i}\right\rangle \bar{u}_{j}\left\langle D_{ \pm}^{j}\right\rangle S_{z}^{i} S_{z}^{j}\right. \\
& +u_{i}\left\langle B_{ \pm}^{i}\right\rangle u_{j}\left\langle B_{ \pm}^{j}\right\rangle S_{x}^{i} S_{x}^{j}-2 u_{i}\left\langle B_{ \pm}^{i}\right\rangle \bar{u}_{j}\left\langle D_{ \pm}^{j}\right\rangle S_{x}^{i} S_{z}^{j} \\
& +2 \mathrm{i} u_{i}\left\langle B_{ \pm}^{i}\right\rangle \bar{u}_{j} \phi_{j} S_{x}^{i} S_{y}^{j}+2 \mathrm{i} u_{i}\left\langle D_{ \pm}^{i}\right\rangle \bar{u}_{j}\left\langle D_{ \pm}^{j}\right\rangle \varphi_{j} S_{y}^{i} S_{z}^{j} \\
& \left.-\mathrm{i} \bar{u}_{i} \bar{u}_{j}\left(\left\langle D_{ \pm}^{j}\right\rangle \phi_{i} S_{y}^{i} S_{z}^{j}+\left\langle D_{ \pm}^{i}\right\rangle \phi_{j} S_{z}^{i} S_{y}^{j}\right)\right\} .
\end{aligned}
$$

The only operators which act nontrivially in the up-down subspace $\{|0,1\rangle,|1,0\rangle\}$ are $S_{z} \otimes \mathbf{1}, \mathbf{1} \otimes S_{z}, S_{x} \otimes S_{x}, S_{x} \otimes S_{y}$, and $S_{y} \otimes S_{x}$. If we define new pseudo-spin operators for pairs $(i, j)$

$$
\begin{aligned}
\sigma_{x}^{p} & =\left|0_{i}, 1_{j}\right\rangle\left\langle 1_{i}, 0_{j}|+| 1_{i}, 0_{j}\right\rangle\left\langle 0_{i}, 1_{j}\right|, \\
\sigma_{y}^{p} & =-\mathrm{i}\left|0_{i}, 1_{j}\right\rangle\left\langle 1_{i}, 0_{j}|+\mathrm{i}| 1_{i}, 0_{j}\right\rangle\left\langle 0_{i}, 1_{j}\right|, \\
\sigma_{z}^{p} & =\left|0_{i}, 1_{j}\right\rangle\left\langle 0_{i}, 1_{j}|-| 1_{i}, 0_{j}\right\rangle\left\langle 1_{i}, 0_{j}\right|,
\end{aligned}
$$

we can project the quoted operators onto the up-down subspace, which yields $S_{z} \otimes \mathbf{1} \rightarrow \sigma_{z}^{p}, \mathbf{1} \otimes S_{z} \rightarrow-\sigma_{z}^{p}$, $S_{x} \otimes S_{x} \rightarrow \sigma_{x}^{p}, S_{x} \otimes S_{y} \rightarrow-\sigma_{y}^{p}$, and $S_{y} \otimes S_{x} \rightarrow \sigma_{y}^{p}$. If we project the Hamiltonian (A.11) accordingly, we find for each pair

$$
\begin{aligned}
H_{\text {pair }}= & -\frac{\Delta_{p}}{2} \sigma_{x}^{p}-\frac{\epsilon_{p}}{2} \sigma_{z}^{p}+\mathrm{i} \Delta_{p} \sigma_{y}^{p} \sum_{q} \frac{2 c_{q}^{p}}{\hbar \omega_{q}}\left(b_{q}-b_{-q}^{\dagger}\right) \\
& +\sum_{q} \hbar \omega_{q} b_{q}^{\dagger} b_{q},
\end{aligned}
$$

with

$$
\begin{aligned}
\Delta_{p} & =J_{i j} \frac{\Delta_{i} \Delta_{j}}{2 E_{i} E_{j}}\left\langle B_{ \pm}^{i}\right\rangle\left\langle B_{ \pm}^{j}\right\rangle \\
\epsilon_{p} & =E_{i}\left\langle D_{ \pm}^{i}\right\rangle-E_{j}\left\langle D_{ \pm}^{j}\right\rangle \\
c_{q}^{p} & =c_{q}^{i}\left(\epsilon_{i} / E_{i}\right)-c_{q}^{j}\left(\epsilon_{j} / E_{j}\right) .
\end{aligned}
$$

Applying Fermi's Golden Rule to the interaction term in (A.15) provides a one-phonon relaxation rate

$$
R_{p}=\alpha \Delta_{p}^{2} E_{p} /\left(2 k_{B}\right)^{3} \operatorname{coth}\left(E_{p} / 2 k_{B} T\right) .
$$

Here, we have used that the oscillating term in $c_{q}^{p} c_{q}^{p *}$ is small so that pairs and single TLS relax approximately with the same coupling parameter $\alpha$ provided that $\epsilon_{i} / E_{i} \approx 1$. Note that because of the coupling of phonons to $\sigma_{y}^{p}$ (instead of $\sigma_{z}^{p}$ ), there is no diagonal coupling term $\propto S_{z}^{p}$ as in (30) after rotation to the pair-TLS eigenbasis.

\section{APPENDIX B:}

In this Appendix we calculate the distribution function for the pair parameters $\Delta_{p}$ and $\epsilon_{p}$ of Eqs. (40) and (41). We note that the single-TLS distribution function for $u=\Delta / E$ and $E=\sqrt{\Delta^{2}+\epsilon^{2}}$ reads $P(E, u)=$ $P_{0} /\left(u \sqrt{1-u^{2}}\right)$. The distribution function for the pair parameter is then given by

$$
\begin{aligned}
P^{(2)}( & \left.\epsilon_{p}, \Delta_{p}\right)=\int \frac{d E_{1}}{1+e^{-\beta E_{1}}} \int \frac{d E_{2}}{1+e^{\beta E_{2}}} \\
& \times \int_{0}^{1} d u_{1} \int_{0}^{1} d u_{2} \int d J P\left(E_{1}, u_{1}\right) P\left(E_{2}, u_{2}\right) P(J) \\
& \times \frac{1}{2}\left\{\delta\left(\epsilon_{p}-E_{1}+E_{2}\right)+\delta\left(\epsilon_{p}+E_{1}-E_{2}\right)\right\} \\
& \times \delta\left(\Delta_{p}-\frac{1}{2} J u_{1} u_{2} e^{-G\left(1-u_{1}^{2}\right) / 2} e^{-G\left(1-u_{2}^{2}\right) / 2}\right) .
\end{aligned}
$$

The factors $\left(1+e^{ \pm \beta E}\right)^{-1}$ account for the thermal occupation of the primary TLS. If the TLS are homogeneously distributed in the glass and interact via a dipolar coupling, $J=U_{0} /|\mathbf{r}|^{3}$, the distribution function of $J$ reads for a three-dimensional probe

$$
P(J)=\frac{4 \pi}{3} \frac{U_{0}}{J^{2}} .
$$

Using now that

$$
\int_{0}^{1} d u \frac{e^{-u^{2} G / 2}}{\sqrt{1-u^{2}}}=\frac{\pi}{2} e^{-G / 4} I_{0}(G / 4),
$$

where $I_{0}(z)$ is a modified Bessel function, and

$\int_{y}^{1} \frac{d x}{(1+x)(1+\mu x)}=\frac{1}{1-\mu}\left[\log \frac{2}{1+y}-\log \frac{1+\mu}{1+\mu y}\right]$,

with $x=e^{-\beta E}, y=e^{-\beta E_{\max }}$, and $\mu=e^{-\beta \epsilon_{p}}$, one easily finds Eqs. (42)-(45).

\section{APPENDIX C:}

In this Appendix we show how a modified tunneling model which comprises an additional Gaussian distribution in the tunneling parameter $\lambda$ centered around the mean value $\lambda_{0} \gg 1$ can provide an algebraic line broadening. The new distribution reads with $\Delta=\hbar \omega_{0} e^{-\lambda}$ :

$$
P(\epsilon, \lambda)=P_{0}+\frac{P_{0} \widetilde{A}}{\sqrt{2 \pi \sigma^{2}}} e^{-\left(\lambda-\lambda_{0}\right)^{2} / 2 \sigma^{2}}
$$

where $\widetilde{A}$ is a dimensionless constant, and $\lambda \geq \lambda_{\min }(E)$, $\lambda_{\min }(E)=\log \left(\hbar \omega_{0} / E\right)$. A similar model has been used previously by Jankowiak and Small27 and by Zimdars and Fayer 32 to discuss 3-pulse photon echo data 33 . 34 A combination of both terms is needed in order that the onset of the algebraic line broadening does not occur too early. In the following it will turn out sufficient to reduce the number of parameters by setting

$$
\sigma^{2} \equiv \lambda_{0} .
$$


The ensuing distribution in $\Delta$ then reads

$$
P(\epsilon, \Delta)=P_{0}\left[\frac{1}{\Delta}+\frac{B\left(\hbar \omega_{0}\right)^{1-\left(1 / 2 \lambda_{0}\right) \log \left(\hbar \omega_{0} / \Delta\right)}}{\Delta^{2-\left(1 / 2 \lambda_{0}\right) \log \left(\hbar \omega_{0} / \Delta\right)}}\right],
$$

where $B=\widetilde{A} e^{-\lambda_{0} / 2} / \sqrt{2 \pi \lambda_{0}}$. This distribution has to be compared with (7). Defining the maximum relaxation rate at given $E$ by $R \equiv e^{-2\left(\lambda-\lambda_{\min }(E)\right)} R_{\max }(E)$, the distribution function in relaxation rates, $R$, and TLSenergies, $E$, reads

$$
(\epsilon / E) P(E, R)=\frac{P_{0}}{2}\left[\frac{1}{R}+\frac{B R_{\max }^{\nu(R)}}{R^{1+\nu(R)}}\right],
$$

where

$$
\nu(R)=\frac{1}{2}-\frac{1}{8 \lambda_{0}} \log \left(R_{\max } / R\right)
$$

From this expression, it is obvious that an algebraic line broadening $\Delta \Gamma(t) \propto t^{\mu}$ with an exponent $\mu<0.5$ occurs for $\lambda_{0} \gg \log \left(R_{\max }(T) t\right)$. The exact calculation reveals

$$
\begin{aligned}
\Delta \Gamma(t)= & \frac{\pi^{2}}{3 \hbar} P_{0}\langle C\rangle k_{B} T\left[\log \left(t / t_{0}\right)\right. \\
+ & \widetilde{A}\left\{\operatorname{erfc}\left(\frac{\lambda_{0}-(1 / 2) \log (K T t)}{\sqrt{2 \lambda_{0}}}\right)\right. \\
& \left.\left.-\operatorname{erfc}\left(\frac{\lambda_{0}-(1 / 2) \log \left(K T t_{0}\right)}{\sqrt{2 \lambda_{0}}}\right)\right\}\right],
\end{aligned}
$$

where $\operatorname{erfc}(x)$ is the complementary error function, and $K \equiv\left(\hbar \omega_{0} / k_{B}\right)^{2} \alpha$. With $\hbar \omega_{0} / k_{B}=O(1 \mathrm{~K})$, one finds $K \approx 10^{10} \mathrm{~K}^{-1} \mathrm{~s}^{-1}$. For $\lambda_{0} \gg \log (K T t)$ this gives an algebraic line growth

$$
\Delta \Gamma(t) \propto B T(K T t)^{(1 / 2)-\left(1 / 8 \lambda_{0}\right) \log (K T t)} .
$$

More details and a comparison to experiment can be found in Ref. 26 .

${ }^{1}$ P. W. Anderson, B. I. Halperin, and C. M. Varma, Philos. Mag. 25, 1 (1972).

${ }^{2}$ W. A. Phillips, J. Low Temp. Phys. 7, 351 (1972).

${ }^{3}$ S. Hunklinger, W. Arnold, S. Stein, R. Nava, and K. Dransfeld, Phys. Lett. A42, 253 (1972).

${ }^{4}$ B. Golding, J. E. Graebner, B. I. Halperin, and R. J. Schutz, Phys. Rev. Lett. 30, 223 (1973).

${ }^{5}$ P. Esquinazi, R. König, and F. Pobell, Z. Phys. B 87, 305 (1992)

${ }^{6}$ J. Classen, Ch. Enss, C. Bechinger, G. Weiss, and S. Hunklinger, Ann. Physik (Leipzig) 3, 315 (1994).

${ }^{7}$ P. Hu and L. R. Walker, Phys. Rev. B 18, 1300 (1978).

${ }^{8}$ J. L. Black and B. I. Halperin, Phys. Rev. B 16, 2879 (1977).
9 T. L. Reinecke, Solid State Commun. 32, 1103 (1979).

${ }^{10}$ Y. S. Bai and M. D. Fayer, Phys. Rev. B 39, 11066 (1989).

${ }^{11}$ H. Maier and D. Haarer, J. Lumin. 64, 87 (1995).

12 (a) H. Maier, B. M. Kharlamov, and D. Haarer, Phys. Rev. Lett. 76, 2085 (1996); (b) H. Maier and D. Haarer, Prag conference proceeding, Elsevier Science; (c) H. Maier, PhDThesis, Bayreuth (1995), unpublished.

13 M. W. Klein, Phys. Rev. Lett. 65, 3017 (1990).

${ }^{14}$ C. C. Yu and A. J. Leggett, Comments Condensed Matter Phys. 14, 231 (1991).

15 S. N. Coppersmith, Phys. Rev. Lett. 67, 2315 (1991).

${ }^{16}$ A. L. Burin and Yu. Kagan, JETP 80, 761 (1995).

${ }^{17}$ K. Kassner and R. J. Silbey, J. Phys. Condens. Matt. 1, 4599 (1989); K. Kassner, Z. Phys. B Condens. Matt. 81, 245 (1990).

18 J. F. Berret and M. Meißner, Z. Phys. B 70, 65 (1988).

${ }^{19}$ L. S. Levitov, Europhys. Lett. 9, 83 (1989); Phys. Rev. Lett. 64, 547 (1990).

${ }^{20}$ G. Hanning, H. Maier, D. Haarer, B. M. Kharlamov Mol. Cryst. Liq. Cryst. 291, 11, (1996).

${ }^{21}$ M. Orrit, J. Bernard, and D. Möbius, Chem. Phys. Lett. 156, 233 (1989).

22 D. W. Pack and M. D. Fayer, Chem. Phys. Lett. 168, 371 (1990).

${ }^{23}$ K. Fritsch, J. Friedrich, and B. M. Kharlamov, J. Chem. Phys. 105, 1798 (1996).

${ }^{24}$ J. Gafert, H. Pschierer, and J. Friedrich, Phys. Rev. Lett. 74, 3704 (1995).

${ }^{25}$ K. Fritsch, J. Friedrich, and J. L. Skinner, Proc. Natl. Acad. Sci. USA 93, 15141 (1996).

${ }^{26}$ A. Heuer and P. Neu, submitted to J. Chem. Phys.

27 (a) R. Jankowiak and G. J. Small, Science 237, 618 (1987); (b) Phys. Rev. B 47, 14805 (1993).

${ }^{28}$ D. Tielbürger, R. Merz, R. Ehrenfels, and S. Hunklinger, Phys. Rev. B 45, 2750 (1992).

${ }^{29}$ S. Rau, C. Enss, S. Hunklinger, P. Neu, and A. Würger, Phys. Rev. B 52, 7179 (1995).

${ }^{30}$ A. Nittke, M. Scherl, P. Esqinazi, W. Lorenz, Junyun Li, and Frank Pobell, J. Low. Temp. Phys. 98, 517 (1995).

${ }^{31}$ B. J. West and M. F. Shlesinger, Int. J. Mod. Phys. 3, 795 (1989).

32 D. Zimdars and M. D. Fayer, J. Chem. Phys. 104, 3865 (1996).

${ }^{33}$ K. A. Littau and M. D. Fayer, Chem. Phys. Lett. 176, 551 (1991)

${ }^{34}$ H. Meijers and D. Wiersma, Phys. Rev. Lett. 68, 381 (1992); J. Chem. Phys. 101, 6927 (1994). 


\section{FIGURE CAPTIONS}

FIG. 1: Coherent up-down coupling between two TLS.

FIG. 2: Energy levels and eigenstates of the Hamiltonian $H_{0, i j}=-(1 / 2)\left(E_{i} S_{z}^{i}+E_{j} S_{z}^{j}\right),\left(E_{i}>E_{j}\right)$. Framed are the up-down states which build a basis for the secondary TLS.

FIG. 3: (a) Hole broadening in PMMA at $1 \mathrm{~K}$ (upper curve) and $0.5 \mathrm{~K}$ (lower curve) compared with the Kassner-Silbey theory for single TLS. The experimental data are from Ref. 12. The two solid lines are $\Delta \Gamma^{(1)}(t)$, Eq. 62 for 1 (upper solid curve) and $0.5 \mathrm{~K}$ (lower solid curve) with $G=32$ $\left(\Theta_{D}=108 \mathrm{~K}\right)$ and $P_{0}\langle C\rangle=6 \times 10^{-5}$. (b) Same figure as above but now with the contribution of both the single and pair TLS [logarithmic and algebraic part of Eq. (61)]. The parameters $P_{0}\langle C\rangle=4 \times 10^{-5}$ and $P_{0} U_{0}=2.5 \times 10^{-6}$ have been optimized to find best agreement with the $0.5 \mathrm{~K}$ data; $G=32$ has been kept fixed. Comparison with the $1 \mathrm{~K}$ data clearly shows that the temperature dependence as predicted by Eqs. (29) and (61) is too strong.

\section{FIG. 1: Neu et al.}
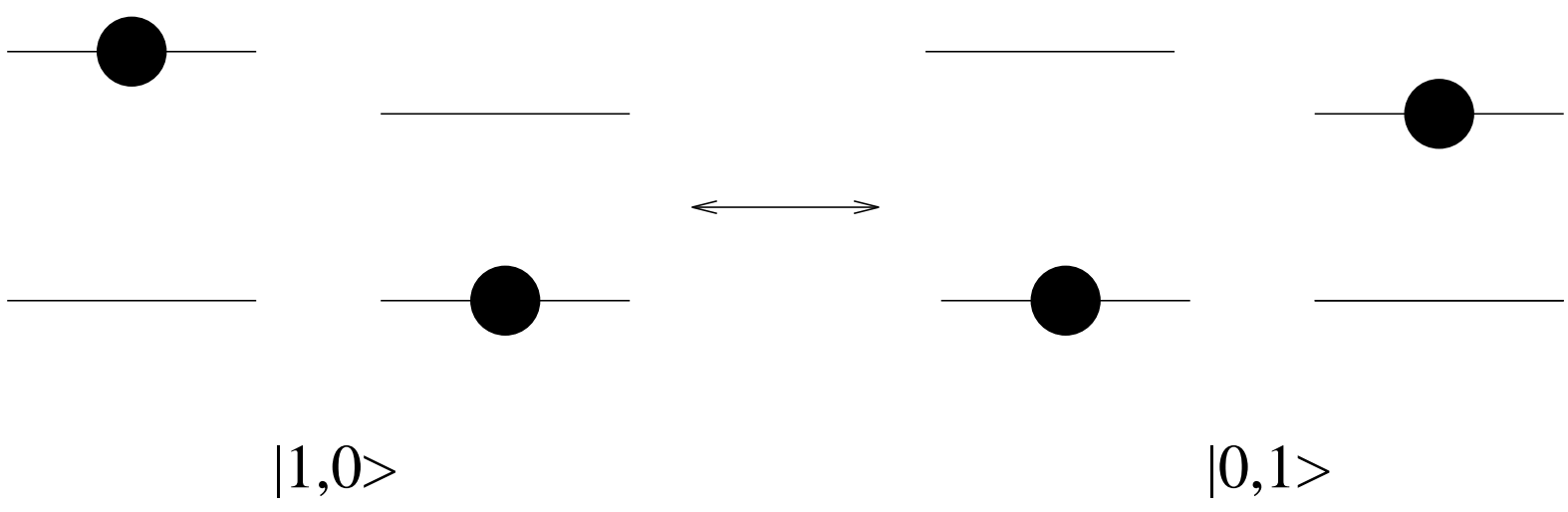

$\mid 0,1>$ 
FIG. 2: Neu et al.

$$
\begin{aligned}
& \left|1_{i}, 1_{j}\right\rangle \quad \frac{1}{2}\left(E_{i}+E_{j}\right) \\
& \text { i - - - - - - - - - - - - - - - } \\
& \text { । }\left|1_{i}, 0_{j}\right\rangle-+\frac{1}{2}\left(E_{i}-E_{j}\right) \text { । } \\
& \text { । }\left|0_{i}, 1_{j}\right\rangle-\frac{1}{2}\left(E_{i}-E_{j}\right) \text { । } \\
& \text { L - - - - - - - - - - - - - J } \\
& \left|0_{i}, 0_{j}\right\rangle \quad-\quad-\frac{1}{2}\left(E_{i}+E_{j}\right)
\end{aligned}
$$


FIG. 3(a): Neu et al.

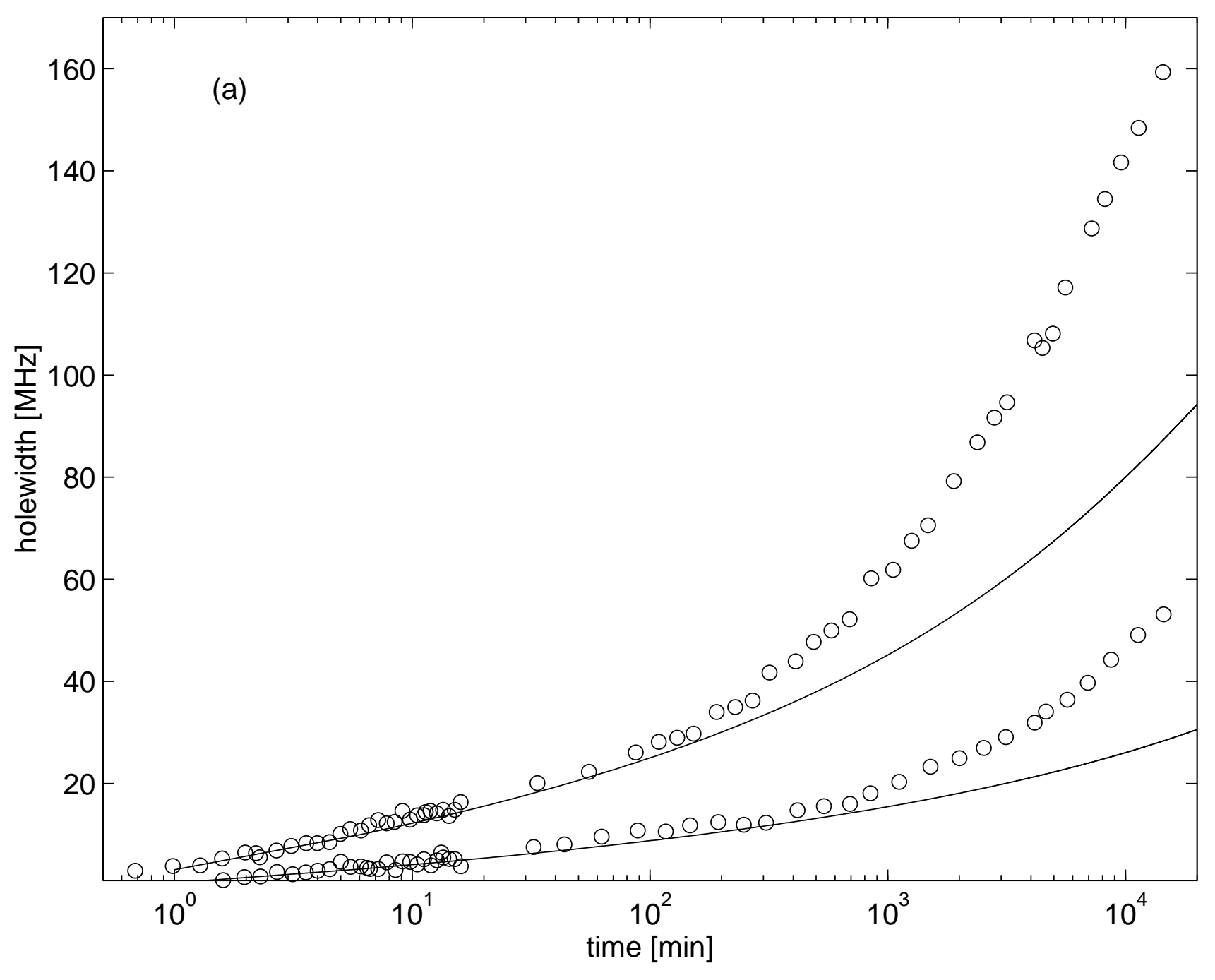


FIG. 3(b): Neu et al.

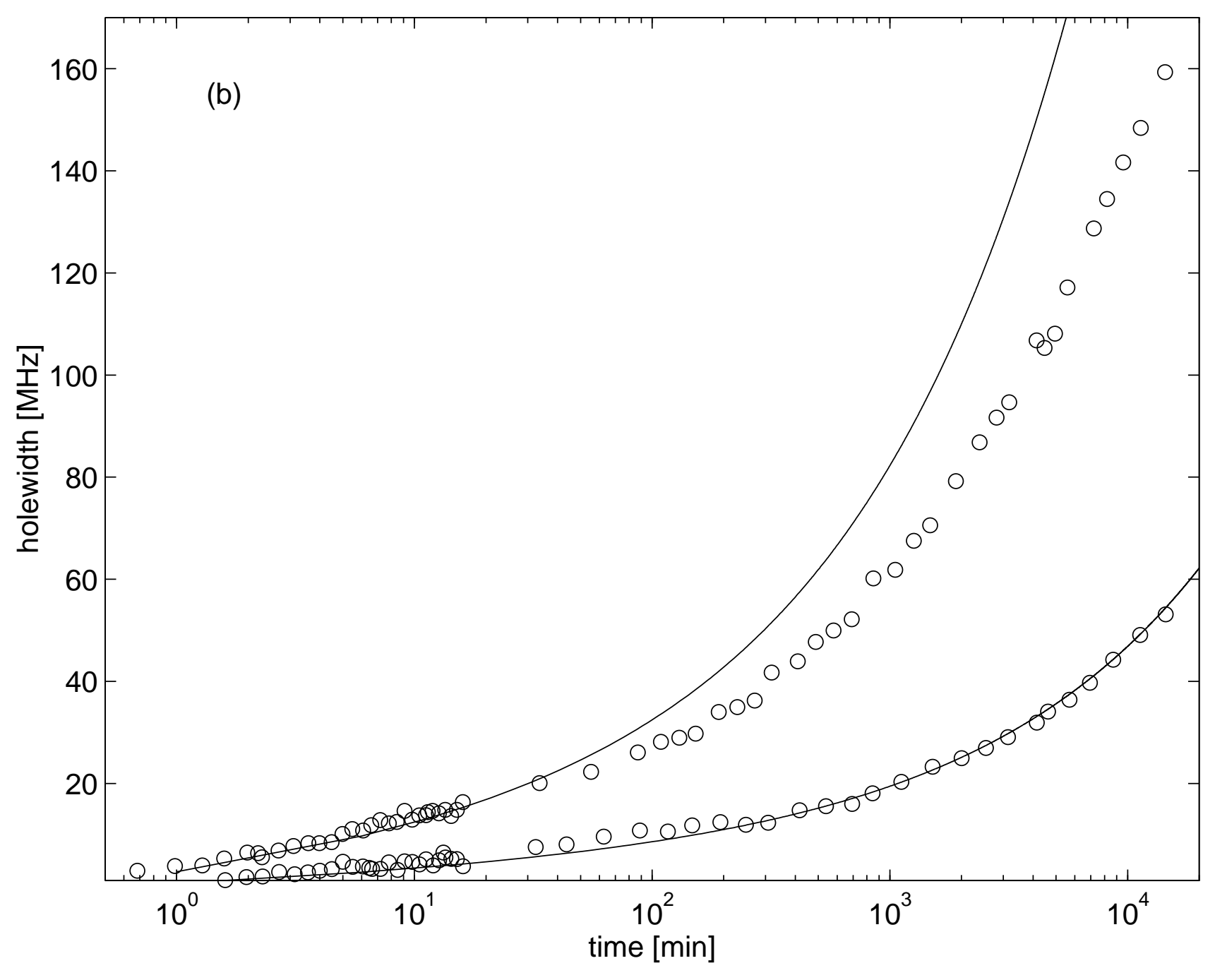

